have been diagnosed with $\mathrm{vCJD}$ or any other form of CJD; donors at increased risk for CJD (eg, those who have received a dura mater transplant or human pituitaryderived growth hormone); donors who have one or more blood relatives diagnosed with CJD; donors who have spent $\geqslant 3$ months cumulatively in the United Kingdom between 1980 and 1996; donors who have spent $\geqslant 5$ years cumulatively in France from 1980 to the present; certain donors who are former or current US military personnel, civilian military personnel, and their dependents; donors who received a transfusion of blood or blood components in the United Kingdom between 1980 and the present; donors who have injected bovine insulin since 1980, unless it can be confirmed that the product was not manufactured after 1980 from United Kingdom cattle; and donors of whole blood or blood components for transfusion and source leukocytes who have lived cumulatively for $\geqslant 5$ years in Europe from 1980 until the present.

FROM: FDA. http://www.fda.gov/cber/gdlns/ cjdvcjd.pdf.

\section{Hygiene and Health: The Epidemiological Link}

Larson and Aiello summarized recent healthcare literature to examine the epidemiological link between personal and household hygiene and the risk of infection. The researchers found 26 interventional and 24 correlational studies during the 20-year period from 1980 to 2000 . On the basis of the literature, the following conclusions were reached. (1) The primary measures associated with reduced incidence of infection include availability of clean water supply, adequate disposal of waste (particularly feces), and hand hygiene. (2) Such interventions are useful primarily for infections spread by the fecal-oral route. (3) In developed countries, the effectiveness of measures such as hand and environmental hygiene has been demonstrated in settings such as schools and day-care centers. (4) In developed countries, the importance of cleanliness in the home environment has received little attention, and the role of the home environment in the spread of infection is unknown. (5) The effectiveness of additional efforts to improve cleanliness (eg, use of antimicrobial products and specific home cleaning regimens) beyond minimal standards has not been demonstrated.
FROM: Larson EL, Aiello AE. Hygiene and health: an epidemiologic link? Am J Infect Control 2001;29:232-238.

\section{Mortality Higher Among Patients Admitted to Hospital on Weekends}

The level of staffing in hospitals often is lower on weekends than on weekdays, despite a presumably consistent day-to-day burden of disease. It is uncertain whether in-hospital mortality rates among patients with serious conditions differ according to whether they are admitted on a weekend or on a weekday. Bell and Redelmeier analyzed all acute-care admissions from emergency departments in Ontario, Canada, between 1988 and 1997 (a total of $3,789,917$ admissions).

The researchers compared in-hospital mortality among patients admitted on a weekend with that among patients admitted on a weekday for three pre-specified diseases: ruptured abdominal aortic aneurysm (5,454 admissions), acute epiglottitis $(1,139)$, and pulmonary embolism $(11,686)$; for three control diseases: myocardial infarction $(160,220)$, intracerebral hemorrhage $(10,987)$, and acute hip fracture $(59,670)$; and for the 100 conditions that were the most common causes of death (accounting for 1,820,885 admissions). Results indicated that weekend admissions were associated with significantly higher in-hospital mortality rates than were weekday admissions among patients with ruptured abdominal aortic aneurysms ( $42 \%$ vs $36 \%, P<.001)$, acute epiglottitis ( $1.7 \%$ vs $0.3 \%, P=0.04$ ), and pulmonary embolism (13\% vs $11 \%, P=.009)$. The differences in mortality persisted for all three diagnoses after adjustment for age, gender, and coexisting disorders. There were no significant differences in mortality between weekday and weekend admissions for the three control diagnoses. Weekend admissions also were associated with significantly higher mortality rates for 23 of the 100 leading causes of death and were not associated with significantly lower mortality rates for any of these conditions.

The authors concluded that patients with some serious medical conditions are more likely to die in the hospital if they are admitted on a weekend than if they are admitted on a weekday.

FROM: Bell CM, Redelmeier DA. Mortality among patients admitted to hospitals on weekends as compared with weekdays. $N$ Engl J Med 2001;345:663-668. 